\title{
DU MASCULIN AU FÉMININ AU MASCULIN : \\ TRANSGRESSIONS IDENTITAIRES ET SEXUELLES DANS \\ SELF DE YANN MARTEL
}

\section{DUMONT, CATHERINE. \\ Candidate à la maitrise en études françaises au Département des lettres et communications de l'Université de Sherbrooke}

Résumé : « Cette fois, ça a commencé par un terrible mal de tête [...] Au matin, le duvet entre mes seins était plus foncé [...] Mes seins se sont aplatis, ma vulve s'est refermée, puis elle a poussé vers l'extérieur » (Martel 269). Ce passage, tiré du roman Self (1998) de Yann Martel, relate la seconde métamorphose que connaîtra le personnage principal au cours du récit. Isabelle Boisclair propose d'appeler «transtextualité » (Boisclair, inédit) ces traversées génériques rendues possible par le seul pouvoir de la littérature. Sachant que les conditions féminine et masculine sont encore aujourd'hui marquées par la « dissymétrie » (Bourdieu 65), nous cherchons, dans cet article, à vérifier si le passage du masculin au féminin (et inversement du féminin au masculin) affecte la condition du personnage. Pour ce faire, notre analyse prendra appui sur les principales théories contemporaines sur le sexe/genre, et ce, dans une perspective à la fois féministe, constructionniste et matérialiste (Delphy; Guillaumin; Wittig).

Mots-clés : Transtextualité, masculin, féminin, déchéance, Yann Martel

\begin{abstract}
Cette fois, ça a commencé par un terrible mal de tête [...] Au matin, le duvet entre mes seins était plus foncé [...] Mes seins se sont aplatis, ma vulve s'est refermée, puis elle a poussé vers l'extérieur" (Martel 269). This excerpt from Yann Martel's novel Self (1998) tells the second transformation that the protagonist will experience during the story. Isabelle Boisclair suggests the word "transtextuality" to refer to such generic crossings made possible by the power of literature (Boisclair, unpublished). Knowing that feminine and masculine conditions are still marked today by asymmetry (Bourdieu 65), we seek, in this article, to verify if the crossing from masculine to feminine (and from feminine to masculine) affects the condition of the character. With this aim in mind, our analysis leans on key contemporary sex-gender theories and does so in a feminist, constructionist and materialist perspective (Delphy; Guillaumin; Wittig).
\end{abstract}

Keywords : Transtextuality, masculin, feminine, decline, Yann Martel 
«Cette fois, ça a commencé par un terrible mal de tête [...] Au matin, le duvet entre mes seins était plus foncé [...] Mes seins se sont aplatis, ma vulve s'est refermée, puis elle a poussé vers l'extérieur » (Martel, 1998 : 269). Ce passage, tiré du roman Self (1998) de Yann Martel, relate la deuxième métamorphose que connaît le personnage principal au cours du récit. Bien que la littérature soit devenue, ces dernières années, un terrain privilégié « de la mise en scène, de l'ambiguïté et de la marginalité sexuelle », qu'en est-il lorsque ni l'opération chirurgicale ni le travestisme ne sont à l'origine du changement de sexe du personnage (St-Hilaire, 1998 : 57)? Tout comme dans Orlando, de Virginia Woolf, nous avons plutôt affaire avec Selfà ce qu'Isabelle Boisclair propose d'appeler un cas de «transtextualité », terme qui, dans le contexte, n'est pas à comprendre au sens où l'entend le théoricien Gérard Genette : plutôt, la « transtextualité » est employée ici pour traduire la façon dont, par le pouvoir de la littérature, le personnage-narrateur transgresse les codes narratifs et les frontières génériques pour se réveiller tantôt femme, tantôt homme. Au sein du roman, on retrouve donc un « je » qui est successivement masculin et féminin et dont les rapports au monde, à l'amour et à la sexualité sont traités sous deux angles différents (Bordeleau, 2004 : 8). Fasciné depuis toujours par le sexe féminin, le narrateur se réveille, le jour de ses 18 ans, en tant que jeune femme. Quelques années plus tard, après avoir été victime d'un viol terrifiant, la narratrice redevient un homme pour «ne plus jamais être aussi vulnérable » (Martel, 1998 : 266).

Soulignons qu'encore aujourd'hui, le statut des femmes est non seulement plus vulnérable, plus précaire, mais également plus globalement désavantagé au sein de la société (Bourdieu, 1998). Aussi, dans le cadre du présent article, nous nous proposons de faire l'examen de chacun des trois épisodes vécus par le personnage, épisodes qui sont balisés par deux changements de sexe. Comme nous savons que les conditions féminine et masculine sont encore marquées par la « dissymétrie ", nous cherchons à vérifier si les changements de sexe opérés par le personnage affectent sa condition (Bourdieu, 1998: 65). Ainsi, ce passage du masculin au féminin (et inversement du féminin au masculin) s'accompagne-t-il d'une déchéance ou d'une ascension? Si oui, sur quels plans s'accomplit-elle? Sur le plan économique, matériel, social ou symbolique? Selon son appartenance au sexe féminin ou masculin, le personnage occupe-t-il une position de dominant ou de dominé? Enfin, à la lumière du nœud « sexe-genre-sexualité », en quoi ce passage brouille-t-il la notion d'identité sexuelle? Afin de bien cerner les enjeux identitaires liés aux deux métamorphoses sexuelles du personnage-narrateur, l'analyse prendra appui sur les principaux concepts entourant les théories contemporaines sur le sexe/genre, et ce, dans une perspective à la fois féministe, constructionniste et matérialiste. Ainsi, les théories de Delphy, Guillaumin et Wittig nourriront notre réflexion.

Roman peu étudié, Self a fait l'objet d'un nombre très limité de recensions critiques. Les recherches entourant la présente étude nous ont toutefois conduits vers un article fort éclairant, écrit conjointement par Isabelle Boisclair et Lori Saint-Martin. Ainsi, "Les conceptions de l'identité sexuelle, le postmodernisme et les textes littéraires », paru en 2006 dans la revue Recherches féministes, démontre en quoi le roman, tant par son discours que par ses procédés textuels, participe à un renouvellement des identités de genre (Boisclair et Saint-Martin, 2006 : 7). Pour cette raison, les deux auteures inscrivent l'œuvre au cœur d'une dynamique postmoderne de l'identité sexuelle, apte à multiplier les reconfigurations sociales et à diversifier les représentations. En ce sens, l'article alimentera plus particulièrement la première partie de notre analyse, alors que nous illustrerons 
en quoi « les nombreuses réflexions [entreprises par le jeune personnage-narrateur] sur l'arbitraire des significations attribuées au masculin et au féminin préparent le terrain » à la façon dont le personnage-narrateur vivra ses métamorphoses (Boisclair et Saint-Martin, 2006 : 18). Dans la seconde partie, nous nous intéresserons au rapport du personnage à la sexualité en regard de ces deux métamorphoses. Enfin, dans la troisième partie, nous scruterons, dans un premier temps, les indices textuels par lesquels des valeurs positives et/ou négatives sont attachées aux genres masculin et féminin afin de déceler si un quelconque statut est valorisé au détriment de l'autre. Enfin, les rapports que le narrateur - devenue narratrice - entretient avec les hommes constitueront un dernier lieu d'analyse.

\title{
LA DÉCOUVERTE DU SYSTÈME DE SEXE/GENRE
}

\author{
UN PORTRAIT QUI OUVRE SUR L'AMBIGUÏTÉ
}

D'entrée de jeu, la couverture de l'édition francophone de Self invite les lecteurs et lectrices à percevoir la problématique identitaire et sexuelle que recèle le roman de Yann Martel. Le choix de l'illustration, Portrait de Friederike Maria d'Egon Schiele, n'est aucunement anodin. Reconnu pour peindre des personnages aux traits déformés, aux positions non conventionnelles et aux corps ambigus qui frôlent l'androgynie, Egon Schiele esquisse une fois de plus un personnage qui nous «laisse dans le doute quant à son identité sexuelle » (Boisclair et Saint-Martin, 2006 : 15). Ce dernier est vêtu d'une robe ample qui dissimule les formes de son corps et d'un bonnet qui recouvre entièrement sa chevelure. Ses traits, plutôt neutres, pourraient, quant à eux, être tout aussi bien ceux d'un homme que d'une femme. Tandis que l'on se tourne vers le titre de l'illustration à la recherche d'une information supplémentaire qui nous permettrait d'accoler un sexe au personnage, nous sommes une fois de plus confrontés à une dualité devant ce prénom : Friederike Maria. Ainsi, cette idée de dualisme permanent est induite dès la première de couverture, puis renforcée tout au long de notre lecture. Le roman soulève une multitude de questions liées à l'identité sexuelle aussi bien qu'à l'identité linguistique : garçon ou fille, homosexuel ou hétérosexuel, anglais ou français (Boisclair et Saint-Martin, 2006 : 13)? Pour le jeune protagoniste de Self, il appert que « la question identitaire ne se résume non pas à un choix (l'un ou l'autre) mais plutôt à un cumul (les deux, voire les trois ou les quatre à la fois ou en succession...) » (Boisclair et Saint-Martin, 2006 : 13).

L'ENFANCE DU NARRATEUR

Le jeune garçon naît avec un esprit libre et grandit auprès de parents « féministes » aux rôles indifférenciés : « Je ne me rappelle pas avoir remarqué, lorsque j'étais petit, une quelconque différence entre mes parents que j'aurais pu attribuer au sexe. Je savais bien qu'ils n'étaient pas une seule et même chose, mais leurs distinctions ne s'exprimaient pas dans des rôles fixes » (Martel, 1998 : 26, 14). Même si Martel met en scène un personnage élevé dans un environnement moderne et ouvert, certaines balises persistent, trahissant le cadre normatif qui sous-tend la bicatégorisation du genre. Bien que ses parents ne lui parlent pas du féminin et du masculin en termes d'opposition, ils les présentent tout de même comme complémentaires: 
Le mâle et la femelle étaient comme la pluie et la terre... Je fus alors frappé en constatant l'étonnante mécanique de l'univers. Imaginez : quelque part au loin, totalement distinct, d'une origine indépendante, existait un organe sexuel adapté au mien, adapté à moi. Je me mis à chercher mon organe sexuel complémentaire, mon véritable amour. (Martel, 1998 : 26)

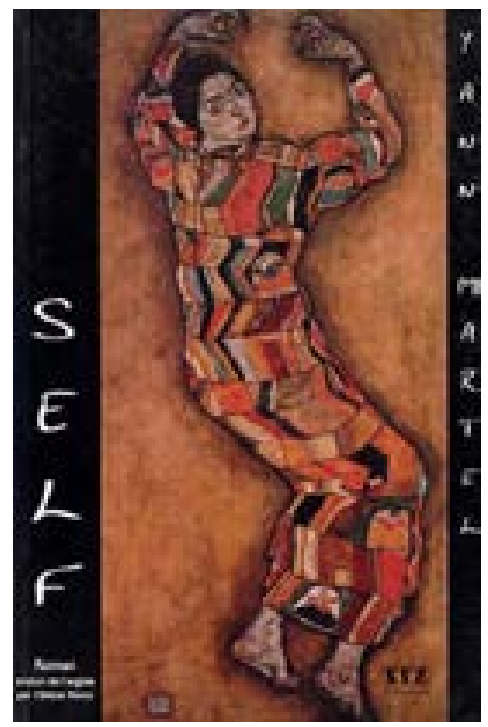

Figure 1. Couverture du roman Self de Yann Martel

C'est ainsi qu'à la maternelle, le narrateur fait la rencontre du jeune Noah et, dans une sorte de naïveté première, en devient amoureux : " Je crois me rappeler que j'aimais la façon de marcher de Noah. II marchait, donc je l'aimais. Un jour, comme ma mère venait me chercher au jardin d'enfants, je l'informai que j'avais trouvé ma future épouse et je pointai fièrement Noah du doigt » (Martel, $1998: 27$ ).

Incapable de déclarer spontanément qu'il aime Noah lorsque sa mère lui demande pourquoi il croit que ce dernier sera sa femme, il lui explique de façon plus concrète que son ami « poss[ède] l'organe sexuel complémentaire du [s]ien » (Martel, 1998 : 27). Toutefois, en voyant un sourire incontrôlable éclairer le visage de sa mère, il « [a] pour la première fois l'intuition que quelque chose lui [a] échappé » et qu'en quittant ce soir-là le jardin, il vient d'une certaine façon de perdre Noah (27). Avant de prendre le chemin du retour, sa mère s'évertue donc à lui donner « des explications rudimentaires sur [sa] personne sexuelle » (Martel, $1998: 27$ ) :

Il n’y avait, en réalité, que deux sexes, et non des quantités infinies. Et ces petits derrières et ces petits doigts que j'avais vus dans les divers exercices de je-te-montrele-mien-si-tu-me-montres-le-tien auxquels j'avais participé étaient les sexes complémentaires en question... (Martel, $1998: 27-28$ )

Bien qu'il n'ait jamais remarqué autre chose que ces deux sexes anatomiques, il avait toujours été persuadé que « cela reflétait simplement la taille réduite de [s]on échantillonnage » et qu'il existait ainsi une multitude de réalités corporelles, au même titre qu'il devait exister des personnes « noires, brunes, jaunes, rouges, bleues, orangées, et peut-être même rayées » (Martel, 1998 : 28) : 
Mais non, insista [s]a mère, il n'y avait que deux sexes. Et, ce qui était encore plus ahurissant, c'est que les petits derrières appartenaient exclusivement aux filles, et les petits doigts exclusivement aux garçons. Les filles, par définition, étaient des femelles avec de petits derrières, et elles seules pouvaient être des épouses. Les garçons, par définition, étaient des mâles avec de petits doigts, et eux seuls pouvaient être des maris. [Il] devai[t] [s]e rappeler ces permutations, car il n'en existait pas d'autres. Non, les épouses ne pouvaient être des garçons. Non, un mari ne pouvait épouser un autre mari. Non, non, non. (Martel, $1998: 28$ )

Au terme de cette discussion, le jeune personnage-narrateur prend soudainement conscience que «les choses [sont] bien plus limitées que [son] esprit ouvert ne les avait imaginées » (Martel, 1998 : 28). Stupéfait de constater que « cette histoire de complémentarité concern[e] seulement un vulgaire point de biologie, une fantaisie anatomique » (Martel, 1998:27-28), il découvre de façon subtile, mais agissante, à quel point le système de sexe/genre est conçu de façon rigide, figée : «Le temps d'une brève balade en voiture, je devins indubitablement un garçon, je découvris une de mes caractéristiques définitives, et l'univers, qui jusqu'alors était une myriade, se divisa en deux camps. J'étais accablé de douleur » (Martel, 1998 : 28). Cet épisode se révèle d'autant plus douloureux qu'il marque en quelque sorte la perte de l'innocence de l'enfant. Pour le personnage-narrateur qui n'a pas encore été totalement socialisé, la vie se déploie devant lui comme un immense terrain de jeu. Les diktats qui gouvernent la sexualité ne sont que des normes sociales contraignantes qui le frappent par leur absurdité.

Comme le soulignent Boisclair et Saint-Martin, c'est « l'arbitraire des assignations identitaires, la pauvreté des possibilités qui révolte le jeune narrateur, le plonge dans le désarroi et le pousse à un questionnement incessant : 'Femelle et mâle? C'est tout? Même sur les autres planètes?' » (cité dans Boisclair et Saint-Martin, 2006 : 19). Non seulement le personnage-narrateur réalise qu'il devra se conformer à un rôle prédéterminé, soit celui dévolu à son sexe, mais aussi apprend-il de façon indirecte ce qu'est l'hétéronormativité, c'est-à-dire l'hétérosexualité pensée comme norme et établie implicitement comme « contrat social » (Wittig, 2007 : 67). En effet, lorsqu'il questionne sa mère afin de savoir s'il peut toujours aimer Noah, cette dernière acquiesce, ajoutant qu'il peut l'aimer autant qu'il le souhaite, car « il est important d'avoir des amis » (Martel, 1998 : 28). Une fois de plus, la sexualité conventionnelle apparaît à ses yeux comme une réalité des plus contraignantes : " J'avais eu la permission d'aimer, et pourtant je sentais - sans pouvoir vraiment expliquer comment - qu'on venait de piéger les océans dans des aquariums » (Martel, 1998 : 28). Toutefois, cet épisode ne l'empêchera pas de dépasser ce système binaire, qu'il considère étouffant, et ce, peu importe si ses collègues de classe « le raill[ent], [l]e bouscul[ent], [l]e terrifi[ent] à cause de [s]es cheveux longs et de [s]on putatif désir des garçons » (Martel, 1998: 58). L'intimidation dont il fait l'objet l'invite d'ailleurs à réfléchir une fois de plus sur le caractère arbitraire des conventions sociales, lesquelles tracent une ligne bien franche entre le masculin et le féminin. Selon lui, d'autres critères se révèleraient davantage aptes à diviser l'humanité :

Un jour, au McDonald's près de l'école, une fois où je me sentais tendu et malheureux, j'étais devant les toilettes avec Sonya, la douce Sonya. HOMMES, disait une porte, FEMMES, disait l'autre. 'Non, pensai-je, non, cela est faux. Ça ne devrait pas être comme ça, ni HOMMES ni FEMMES. Ça devrait être AMIS et ENNEMIS. Ce devrait être la division naturelle des choses, ce serait un meilleur reflet de la réalité.' De cette façon, Sonya et moi nous pourrions entrer par une porte, et les autres, par l'autre porte. (Martel, $1998: 61$ ) 
Bien que le sexe constitue un trait déterminant dans la société, pour lui, il n'aura, « dans le domaine de l'amour, [...] pas plus d'importance que les parfums quand il s'agit de crème glacée » (58). Dès sa tendre enfance, le narrateur privilégie plutôt « le cumul des identités sexuelles généralement considérées comme opposées » et la multiplicité au détriment de l'unicité (Boisclair et Saint-Martin, 2006 : 15). La découverte d'un ver de terre le plongera d'ailleurs dans un état d'euphorie incroyable, alors que ce dernier incarnera à ses yeux une sorte d'« idéal » par sa nature hermaphrodite :

J'examinai de près cette suprême créature brune pendant qu'elle se contorsionnait mollement entre mes doigts. Mâle et femelle! C'était extraordinaire. [...] Je m'éloignai avec ce miracle de l'univers. Chaque fois que les mots me revenaient - 'À la fois mâle et femelle!' -, j'étais de nouveau ébahi. Si Dieu existait - ? -, Il, Elle, devait sûrement avoir la tête chauve et arrondie d'un verre de terre. (Martel, 1998: 32)

Pour Boisclair et Saint-Martin, il appert que Yann Martel, par le truchement de son jeune narrateur,

prône l'existence et l'acceptation sociale d'identités fluides, changeantes et multiples. On voudrait non pas deux possibilités mais un nombre infini de permutations susceptibles de changer au gré du temps, des rencontres et de l'évolution personnelle. Or, les événements donneront raison au jeune narrateur puisque lui-même changera non seulement d'orientation sexuelle, ce qui est tout de même plus courant, mais aussi - et sans effort de sa part - de sexe. (Boisclair et Saint-Martin, 2006 : 15)

VERS UNE PLUS GRANDE MULTIPLICITÉ

Comme nous l'avons souligné précédemment, les deux transformations sexuelles du personnage-narrateur sont le résultat de la transtextualité, procédé par lequel Yann Martel transgresse les codes du réalisme pour faire de son personnage un être qui se métamorphose de façon inexplicable - et qui reste inexpliquée. D'abord, certains symptômes, dont « un changement de voix vers le plus aigu », " une légère douleur aux hanches » et « la disparition de [s]on acné » apparaissent, puis annoncent la première métamorphose du personnage, qui est complétée au cours d'une nuit particulière (Martel, 1998 : 94) :

Je me suis réveillée soudainement. Je ne sais pas pourquoi ni à quoi je rêvais. Je me suis dressée. Tout était confus. Je ne me souvenais de rien, ni de mon nom, ni de mon âge, ni où j'étais. L'amnésie totale. Je savais que je pensais en français, ça au moins c'était sûr. Mon identité était liée à la langue française. Et je savais aussi que j'étais une femme. (Martel, 1998 : 96)

Cette première transformation, qui coïncide avec le passage à l'âge adulte du personnage-narrateur ${ }^{1}$, de même que la seconde, qui survient quelques années plus tard, lui permettent d'envisager sa sexualité en termes de possibilités et de multiplicité. Au lieu d'entretenir un rapport limité et contraignant au monde, il possède un plus grand éventail de choix en ce qui a trait à ses relations et peut, du coup, expérimenter la sexualité d'un point de vue à la fois masculin et féminin. II devient donc une femme qui est intime avec des femmes, puis une femme qui se lie avec des hommes,

1. Dans La pensée straight, Monique Wittig nous invite à considérer la catégorie de sexe comme " une catégorie politique qui fonde la société en tant qu'hétérosexuelle » (2007 : 38). Plus précisément, elle perçoit le primat de la « différence des sexes » comme une façon de réguler le genre et d'ainsi maintenir l'ordre hétérosexuel fondé sur un rapport d'appropriation des femmes par les hommes. Le projet de Wittig consiste d'ailleurs à «traquer » ce « cela-vade-soi hétérosexuel » pour ainsi rompre le contrat social hétérosexuel (Wittig, 2007 : 60, 69). 
pour ensuite redevenir un homme qui couche avec des hommes et, enfin, un homme qui a des relations sexuelles avec des femmes. Ainsi, il réfute sa thèse d'enfance selon laquelle les choix sexuels seraient comparables à un restaurant où il n'y aurait que deux plats et où l'on saurait d'avance lequel nous serait destiné (Martel, 1998 : 19). Néanmoins, bien que le personnage-narrateur soit né avec un esprit libre, il a quand même incorporé certains des schèmes mentaux qui régissent notre société. À la suite de sa première transformation, lorsqu'il vit pour la première fois, en tant que femme, une relation avec un homme, la pensée hétéronormative est encore à l'œuvre :

C'est un homme, ceci est de l'homosexualité, je suis un homosexuel. C'est la pensée qui m'avait traversé l'esprit dans le salon lorsque Tom avait embrassé le sommet de ma tête [...] C'est fou, je le sais bien. Nous étions en train d'accomplir un acte hétérosexuel parfaitement normal, et même banal, mais ça revenait, encore et encore, c'est un homme. Ceciest de l'homosexualité, je suis un homosexuel, même si cette impression de faire quelque chose d'interdit n'interdisait rien... (Martel, 1998 : 173-74)

Il en sera de même à la toute fin du récit, lorsque la narratrice, redevenue homme, expérimentera une relation avec une femme et déclarera : "J'étais une lesbienne tiède » (Martel, 1998 : 281). Ces observations trahissent la persistance du cadre hétéronormatif en regard duquel toutes les relations sont qualifiées. Si le cumul identitaire est d'abord perçu par le narrateur comme une expérience jubilatoire, il semblerait toutefois, à mesure que le récit progresse, que les deux métamorphoses s'accompagnent aussi d'une grande désillusion.

\section{LE MASCULIN/FÉMININ COMME RAPPORT DE POUVOIR}

\section{L'IDÉALISATION DU FÉMININ}

Bien avant sa première métamorphose, le jeune personnage-narrateur, cherchant à démystifier les normes du genre, se tourne vers la langue française, convaincu que cette dernière parviendra à l'éclairer sur le sujet en lui donnant « le sexe de tout ce qui exist[e] » sur la terre (Martel, 1998 : 59). À travers son interprétation du langage, on décèle rapidement un parti pris du jeune garçon en faveur d'un genre en particulier : il attribue aux termes féminins des valeurs positives qui trouvent leur pendant négatif du côté des termes masculins. De ce fait, « [il] [accept[e] volontiers que les camions et les meurtres soient masculins tandis que les bicyclettes et la vie [sont] féminines » (Martel, 1998 : 59). Mais ces découvertes ne lui apportent qu'une satisfaction passagère.

En effet, il ne trouve « pas très logique que les ordures soient féminines et que le parfum soit masculin, et pas logique du tout que la télévision, qu'[il] aurai[t] jugée répugnante et masculine, soit en réalité féminine » (Martel, 1998 : 59). Cette idéalisation du féminin qui, soulignons-le, constitue un renversement des normes habituelles, se perpétue aussi à l'adolescence, alors qu'il tente de percer les secrets de la sexualité féminine aux côtés de son amie Sonya. Les menstruations provoquent chez lui « une source d'émerveillement profond » (Martel, 1998: 62) et « même si les filles ne sembl[ent] pas considérer le cycle menstruel comme quelque chose de joyeux, et sûrement jamais comme une expérience transcendantale » (Martel, 1998: 61), il ne cesse d'en être fasciné. Contre toute attente, à la suite de sa métamorphose en femme, ses menstruations sont vécues comme une expérience traumatisante: 
Plusieurs mois passèrent avant que j'aie mes premières règles. La vision exaltée que j'avais du cycle menstruel se trouva considérablement assombrie le matin où je me réveillai dans des draps ensanglantés après avoir passé une nuit perturbée par la fièvre, la migraine, la nausée. [...] Je fus horrifiée, en état de choc. [...] Ce désordre, cette saleté, cette odeur fétide, cette douleur... une fois par mois! C'est injuste, cosmiquement injuste! (Martel, 1998 : 105)

Ce passage marquera d'ailleurs le début d'une longue série d'événements qui feront tour à tour perdre à la personnage-narratrice une bonne partie de ses illusions quant à son nouveau statut.

LA DOMINATION MASCULINE

Pendant son enfance, le personnage-narrateur, après avoir saisi l'existence du système binaire, constate bientôt qu'il existe une hiérarchie entre les sexes, réflexion qu'il se fait alors qu'il déambule dans les couloirs du Parlement:

'C'est le parlement, masculin. C'est le pouvoir.' Je retournais chez moi à la maison, féminin, où j'irais peut-être à ma chambre, la chambre, où je m'installerais pour lire un livre, masculin, jusqu'à l’heure du dîner. [...] Après ce jour masculin dur et productif, je me reposerais pendant la nuit féminine. (Martel, 1998 : 59)

On remarque que le masculin et le féminin sont représentés de façon dichotomique, alors que sont opposés la sphère du public à celle du privé, l'actif au passif, le domaine de l'esprit à celui du corps indolent. Bien que le personnage-narrateur comprenne indirectement que le système de sexe/genre superpose des oppositions sur les plans social et symbolique, jamais les effets de la domination masculine ne lui étaient apparus sous une forme aussi violente avant de s'être véritablement mis dans la peau d'une femme :

Où les hommes se sentent-ils mal à l'aise? Dans des ascenseurs, [...] des jacuzzis, la nuit, sur... des quais de métro déserts, [...] des taxis..., dans des universités, au Vatican, dans des westerns... ? Non. Dans aucun de ces endroits, à aucune de ces occasions, on ne dira à un homme qu'il n'est pas le bienvenu parce qu'il est un homme. (Martel, $1998: 138-9)$

Au cours d'un voyage en Turquie, cette impression de se sentir « autres » simplement parce qu'elles sont des femmes se fait encore plus lourdement ressentir par la personnage-narratrice et par Ruth, sa compagne de voyage :

Il était parfois difficile de voyager en Turquie. Il y avait des tracasseries. [...] Mais lorsque j'y repense maintenant, certains de ces ennuis étaient inacceptables. Ils avaient un lien commun : les hommes. Les hommes qui nous regardaient ouvertement de haut en bas. Les hommes qui se mettaient à sourire en nous voyant et qui se tournaient vers leurs amis en nous désignant d'un hochement de tête. Les hommes qui se frottaient contre nous pour nous dépasser dans les rues qui n'étaient pas bondées, et qui passaient leurs mains sur nos seins. (Martel, $1998: 140$ )

Chacun de ces gestes constitue une agression à l'endroit des deux voyageuses, agression qui repose sur un rapport d'appropriation. Ainsi que le suggère Colette Guillaumin dans son étude sur le racisme: 
On ne prend publiquement que ce qui vous appartient ; même les kleptomanes les plus débridés se cachent pour tenter de saisir ce qui n'est pas à eux. Pour les femmes, c'est inutile de se cacher. Elles sont un bien commun. [...] La publicité même de cette mainmise, le fait qu'elle revête aux yeux de beaucoup, et en tous cas des hommes dans leur ensemble, un tel caractère de 'naturel', de quasi 'allant de soi', est l'une de ces expressions quotidiennes et violentes de la matérialité de l'appropriation de la classe des femmes par la classe des hommes. (Guillaumin, 1992 : 13)

Objet de désir, le corps des deux femmes est soumis impunément au regard des hommes, phénomène qui est non sans rappeler l'épisode où, enfant, le narrateur-garçon contemple un livre destiné à la sexualité des adolescents et regrette qu'« il n'y ait pas une seule photo de pénis en érection, d'adolescent ou d'adulte », tandis que la nudité féminine y est dévoilée (Martel, 1998: 66). Si le corps féminin est dénudé, érotisé, prêt à être « consommé » par la gent masculine, la femme n'a quant à elle ni le privilège ni le droit de détailler le corps masculin. Quoi qu'il en soit, au terme du périple, les deux femmes deviennent de véritables "vétérans du voyage de combat », bien résolues à ce qu'« aucun Pierre Jean Jacques turc ne déconn[ent] avec [elles]» (Martel, 1998 : 140). L'armure qu'elles parviennent à se forger comporte toutefois des failles et la narratrice le soulève bien : « Nous n'étions pas des chars d'assaut. Nous étions deux femmes portant des vêtements de coton, [...] deux femmes qui voyageaient dans un grand pays mâle » (Martel, 1998 : 140). Enfin, certaines inégalités révoltent au plus haut point la personnage-narratrice, si bien qu'elle se jure, à la fin du voyage, «qu'aucun homme n'aurait jamais le contrôle sur [elle] » (Martel, 1998: 142). Mais la réalité la rattrape bien assez vite, faisant voler en éclats cette promesse faite quelques années plus tôt.

\section{UNE CHUTE INÉVITABLE}

Vers la fin du récit, la narratrice subit un viol au terme duquel elle a l'impression d'avoir été « anéanti[e] » par son agresseur (Martel, 1998 : 273). Ainsi, « en une fraction de seconde, les choses ont basculé du normal à l'épouvantable » pour la jeune femme, et ce, sans qu'il n'y ait eu " aucun avertissement » (Martel, 1998 : 244). Son voisin a pénétré dans son appartement, sous prétexte de vouloir y jeter un coup d'œil, a refermé la porte, puis a tout simplement ordonné à la narratrice de se déshabiller. Les choses se sont ensuite enchaînées de façon rapide et confuse. La détresse qui envahit la personnage-narratrice est d'ailleurs accentuée par la mise en page du texte. De la page deux cent quarante-quatre à la page deux cent soixante-trois, le texte est divisé en deux colonnes : la première relate les événements qui se sont déroulés lors du viol, tandis que la seconde contient une longue série de points de suspension entre lesquels s'immiscent et s'accumulent les mots « terreur » et « douleur ». Les points de suspension et les blancs typographiques symbolisent aussi bien la paralysie de la pensée que la difficulté à dire et à représenter cette violence lorsqu'elle est vécue de l'intérieur, seules les émotions affluant à la perception. Ces « vingt minutes » que la personnage-narratrice compare à un long « assaut » ayant « duré des heures », ont pour effet de la « désubjectiver», de la déshumaniser, de la tuer à petit feu (Martel, 1998 : 244, 144, 244) : 
À ses yeux à lui, je n'étais digne d'aucun respect. J'étais réduite à rien, mon être, mes sentiments étaient volontairement méprisés. On ne peut imaginer combien il est difficile de remonter la pente après un tel avilissement. On glisse constamment vers le bas. [...] Je ne sais pas pourquoi on appelle ça un viol. Pour moi, ça a été un meurtre. J'ai été tuée ce jour-là et, depuis, je dois traîner la mort en moi, une grisaille qui vagabonde dans mon intérieur coloré ; parfois, c'est mon estomac qui est mort, parfois, c'est ma tête, parfois, ce sont mes intestins, souvent c'est mon cœur. (Martel, 1998:271)

À la suite de ces événements, elle choisit donc de « quitter Montréal - quitt[er] sa vie - de façon brutale et désordonnée » (Martel, 1998 : 270). Songeant au suicide, elle se coupe les cheveux, geste qui annonce sa deuxième métamorphose, celle au cours de laquelle elle redeviendra un homme, pour «ne plus jamais être aussi vulnérable. Jamais » (Martel, 1998 : 266). Si la première transformation s'était déroulée en douceur, de façon presque imperceptible, la seconde s'effectue difficilement et douloureusement :

Cette fois, ça a commencé par un terrible mal de tête. J'avais si mal que je pensais que mon crâne allait éclater. [...] Mon poil poussait en me donnant des démangeaisons et je me suis gratté la poitrine et les jambes jusqu'au sang, mais c'était une douleur que je m'infligeais moi-même, tout comme le dégoût que je ressentais à voir émerger mon pénis en était un autre. (Martel, 1998 : 269)

Traumatisé par son expérience et alimentant une haine certaine envers les hommes, le narrateur ne peut ressentir que du mépris face à son sexe masculin. Pourtant, cette transformation se présente à lui comme la seule façon de se sentir à nouveau « plus fort, plus dur » et « invincible » (Martel, $1998:$ 276, 277).

Un jour, ruant de coups un homme ivre dans une ruelle, il profite de son nouveau statut de mâle pour se venger symboliquement contre tous les hommes, mais aussi contre « toute la race humaine » (Martel, 1998 : 277). Sa rencontre avec Cathy lui permettra toutefois de se réconcilier avec la vie, alors que cette relation se transformera en une véritable " expérience de la fusion sexuelle » (Boisclair et Saint-Martin, 2006 : 23). En effet, à l'occasion d'un voyage en Thaillande, le narrateur, songeant qu'il n'a pas de seins, s'allonge auprès de Cathy pour ainsi sentir la poitrine de sa compagne dans son propre dos: "Je me suis collé encore davantage et ses seins m'ont traversé - j'avais [maintenant] des seins » (Martel, 1998 : 282). Comme le soutiennent Boisclair et Saint-Martin, «ce qu'on voit ici, ce sont donc non pas des formes exclusives - être homme OU femme - mais le cumul : être homme ET avoir des seins. L'idéal, en quelque sorte, serait l'ouverture, la multiplicité » (Boisclair et Saint-Martin, 2006 : 23).

\section{CONCLUSION}

À la lumière de notre analyse, Self de Yann Martel est un roman qui trouble, sinon qui questionne, nos définitions et nos conceptions imaginaires les plus usuelles concernant la notion d'identité sexuelle. Sur un mode humoristique, Martel met dans la bouche de son narrateur-enfant une multitude d'interrogations qui frappent le lecteur et la lectrice autant par leur amusante naïveté que par leur surprenante lucidité. Étonné d'abord, révolté ensuite, par le caractère arbitraire et restrictif des conventions sexuelles, le garçon aspire indirectement à vivre dans un monde où le sexe, le genre et 
I'orientation sexuelle ne seront plus perçus comme des traits discriminants (Grange, 2010 : 111). II nous invite par ailleurs à concevoir la vie et la sexualité en termes de choix et de possibilités, principe qu'il incarne lui-même en changeant de sexe à deux reprises. Rappelons que la narration, assumée au départ par un « je » masculin, change radicalement pour ensuite être prise en charge par un « je » féminin. Si, de prime abord, ces métamorphoses semblent receler un caractère jubilatoire, il semble toutefois que le/la narrateur/narratrice peine à se détacher des assignations normatives indissociablement liées à une hétérosexualité obligatoire.

Mais encore, le caractère négatif associé à l'épisode des menstruations, à celui du voyage en Turquie et à celui du viol a tôt fait de désillusionner le personnage principal quant à sa condition féminine. Par ailleurs, on ne peut que prendre acte de la déchéance à la fois sociale et symbolique qui accompagne le passage du masculin au féminin, déchéance à laquelle l'instance auctoriale mettra un terme en transformant le personnage en homme à la toute fin du récit. Retenons que l'expérience féminine et l'expérience masculine du protagoniste lui octroient donc des statuts fluctuants : aussi bien dire que le personnage éprouve de l'intérieur la politique des identités. II occupe une position dominante lorsqu'il performe le genre masculin et il est relégué à la position du dominé lorsqu'il performe le genre féminin. Le caractère, la personnalité et les aspirations du personnage, quant à eux, demeurent sensiblement les mêmes, ce qui suggère que sans les constructions sociales qui l'entourent, le sexe n'a qu'une incidence minime sur le façonnement de notre identité.

La dernière phrase du roman soulève d'ailleurs une interrogation intéressante quant à l'identité sexuelle finale du personnage : « J'ai trente ans. Je pèse soixante-trois kilos. Je mesure un mètre soixante et onze. J'ai les cheveux bruns et bouclés. Mes yeux sont gris-bleu. Mon groupe sanguin est O positif. Je suis d'origine canadienne. Je parle le français et l'anglais » (Martel, 1998 : 183). Soulignons la façon dont l'identité sexuelle est manifestement passée sous silence, alors même qu'elle est habituellement mise à l'avant-plan : l'identité se construit autour d'une série de traits comme l'âge, la langue et la nationalité, tandis que la dernière phrase du roman s'articule de façon à ce que le genre ne puisse être décelé. La transformation du personnage en homme est-elle définitive? Nous l'ignorons. Et Martel nous invite à conclure notre lecture en songeant qu'après tout, cela n'a aucune importance. 
Boisclair, Isabelle et Lori Saint-Martin. «Les conceptions de l'identité sexuelle, le postmodernisme et les textes littéraires. » Recherches féministes 19.2 (2006) : 5-27. Imprimé.

Bordeleau, Francine. "Yann Martel, l'écrivain hors frontières. » Lettres québécoises 113 (2004) : 8-10. Imprimé.

Bourdieu, Pierre. La domination masculine. Paris : Seuil, 1998. Imprimé.

Delphy, Christine. L'Ennemi principal. Économie politique du patriarcat. Paris : Syllepses, 1998. Imprimé.

Grange, Juliette. «Genre et sexe : nouvelles catégories épistémologiques des sciences humaines. » Cités : Philosophie, Politique, Histoire. Dir. Michel Prigent. Paris : Presses universitaires de France, 2010. 107-21. Imprimé.

Guillaumin, Colette. «L Le catégorisant. »L'idéologie raciste. 1972. Paris: Gallimard, 2002. 291- 301. Imprimé.

---. Sexe, race et pratique du pouvoir. L'idée de nature. Paris : Côté-femmes, 1992. Imprimé.

Martel, Yann. Self. Montréal : XYZ, 1998. Imprimé.

Saint-Hilaire, Colette. «Crise et mutation du dispositif de la différence des sexes : regard sociologique sur l'éclatement de la catégorie sexe. » Les Limites de l'identité sexuelle. Dir. Diane Lamoureux. Montréal : Remue-ménage, 1998. 57-85. Imprimé.

Wittig, Monique. La pensée straight. 2001. Paris : Éditions Amsterdam, 2007. Imprimé. 